\title{
The UK food industry's views on front of pack nutrition labelling: a quantitative study
}

\author{
S. Howlett ${ }^{1}$ and O. B. Kennedy ${ }^{1}$ \\ ${ }^{1}$ Department of Food and Nutritional Sciences, University of Reading, UK
}

\begin{abstract}
Nutrition labels are seen as important in providing dietary information to help consumers make informed healthy food choices ${ }^{(1)}$. Labels may be the only source of nutritional information available to the consumer at the point of purchase, therefore it is important the information is easy to find, read, interpret and understand ${ }^{(2)}$. The desire of UK retailers to display their nutrition labelling information in a consumer friendly and appealing manner lead to the development of the front of pack label also called signposting ${ }^{(3)}$. Several different retailer and manufacturer schemes have emerged, which all share the aim of increasing the use and understanding of nutritional information to help consumers make healthy and informed choices. In June 2010, the European Parliament backed a proposal for more uniform food labelling in the European Union (EU). They rejected the traffic light colour coding system, opting instead for GDA for front of pack nutrition labelling ${ }^{(4)}$ which is in line with the majority of current food industry practice in the UK and Europe ${ }^{(5)}$. It is reported that this choice of label by the food industry is based on evidence from their in-house customer research; however, they are under no obligation to make this information available and the existing academic literature contains little insight into the industries view of nutritional labelling formats. The current study has sought to address this knowledge gap and has used quantitative research to investigate the views of food professionals, especially nutritionists $(\mathrm{N})$ and food technologists (FT), from national retailers, food manufacturers and other interested stakeholders on both current and proposed front of pack nutrition labelling options in the UK and Europe.

The questionnaire developed contained five sections: views on current front of pack (FOP) nutrition labelling; awareness of the introduction of mandatory FOP labelling in the UK and Europe; the financial implications of introducing FOP labels; improvements to current nutritional labelling and demographical information. It consisted of twenty eight attitudinal questions, containing both closed and open-ended questions. It was sent out in August 2010 via e-mail to 300 selected participants using the Bristol Online Survey (BOS) tool and was open for 4 weeks; the response rate was $28 \%$.

Of these, $56 \%$ of respondents agreed with the introduction of mandatory FOP nutrition labelling compared to $27.4 \%$ who disagreed. Views on the FSA's standardised and integrated label format as the best way forward for UK consumers were split, with $40.5 \%$ against and $36.9 \%$ in favour. $46.5 \%$ of respondents agreed with the rejection of mandatory EU traffic lights while $28.6 \%$ disagreed. However, $59.6 \%$ agreed with the use of voluntary traffic lights across Europe. 36.9\% of respondents favoured the GDA in monochrome format for future use by the UK food industry, with $43 \%$ of respondents currently using this label. Views differed according to job role, with $77 \%$ of $\mathrm{N}$ agreeing with mandatory FOP labels $v .40 \% \mathrm{FT}$, and with $40 \%$ of FT agreeing with the proposal that traffic light labels become the legal FOP format for Europe be rejected v. $3 \%$ of $\mathrm{N}$.

Overall respondents from UK food industry favoured monochrome guideline daily amounts as the current and future FOP nutrition labelling format. This is in line with the European Union's plans for mandatory nutrition labelling. Traffic lights, although preferred by consumers, were viewed as a voluntary addition. However, the study highlights differences in views of nutritionists and food technologists, key stakeholders who influence decision making through company policies on product innovation and reformulation.
\end{abstract}

1. Grunert KG \& Wills JM (2007) J Public Health 15, 385-399.

2. Mackison D, Wrieden WL \& Anderson AS (2010) E J Clin Nutr 64, 210-217.

3. BRC (2009) http://www.brc.org.uk/acommitmenttohealth (accessed July 2010).

4. BBC (2010) http://www.bbc.co.uk/news/10327312 (accessed July 2010).

5. CIAA (2010) http://www.ciaa.be/asp/index.asp (accessed July 2010). 